\title{
Perinatale Outcomes nach Gabe von Tamsulosin in der Schwangerschaft
}

\section{Die Behandlung einer symptomatischen Urolithiasis in der} Schwangerschaft kann sich schwierig gestalten, nur knapp die Hälfte der Steine geht spontan ab. Die Gabe des $\alpha$-RezeptorAntagonisten Tamsulosin soll die Steinaustreibungsrate erhöhen, allerdings gehört das Medikament nach FDA-Einteilung zur Klasse B (kein Hinweis auf Risiken für den Fetus im Tiermodell, Studien zum Risiko beim Menschen liegen nicht vor). Mediziner der Mayo Clinic haben sich der Frage angenommen, ob die Gabe von Tamsulosin während der Schwangeschaft einen negativen Einfluss haben kann. J Urol 2016; 195: 99-103

mit Kommentar

Die Gabe von Tamsulosin bei Schwangeren scheint nicht mit dem vermehrten Auftreten ungünstiger perinataler Outcomes verbunden zu sein. Zu diesem Schluss kommen George Bailey und seine Kollegen in ihrer retrospektiven Auswertung.

Die Wissenschaftler hatten zunächst in Krankenakten insgesamt 27 Schwangere identifiziert, die zwischen 2000 und 2013 trotz bestehender Schwangerschaft Tamsulosin zur Behandlung einer Urolithiasis

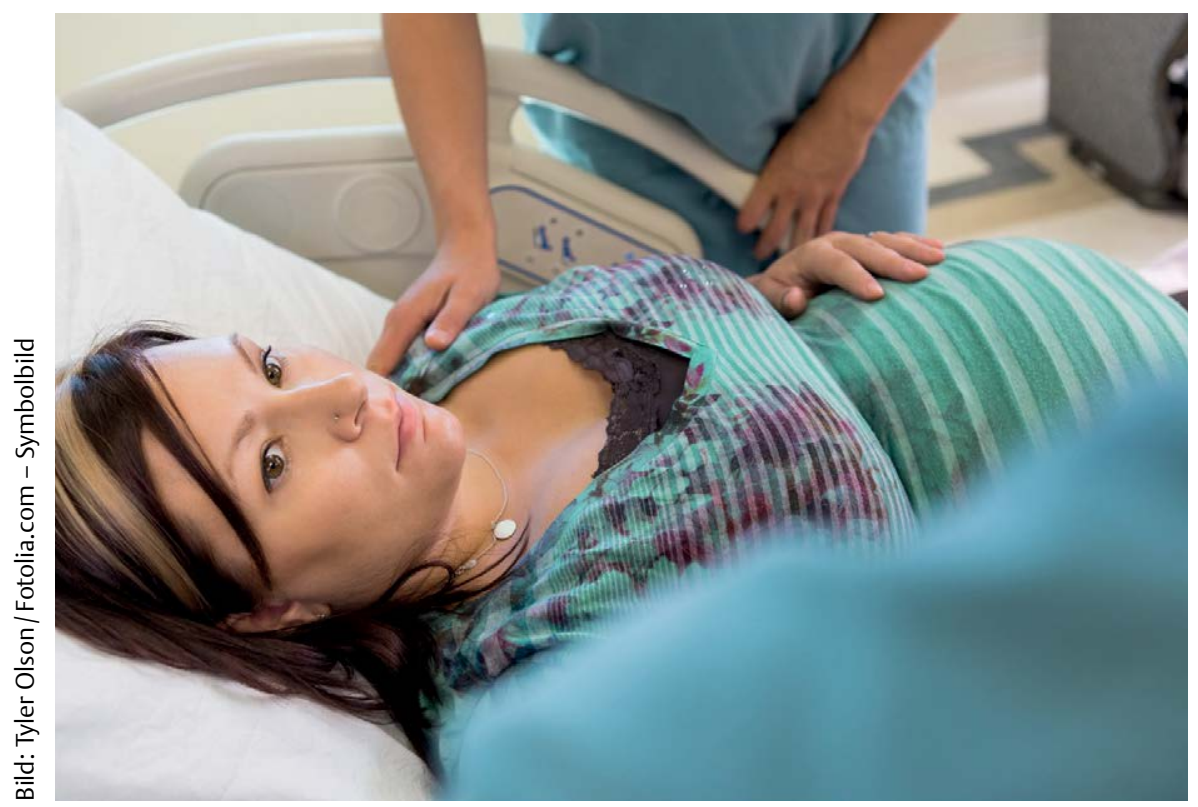

gar-Werte, Notwenigkeit einer Aufnahme auf die Neugeborenen-Intensivstation und die perinatale Sterblichkeit.

\section{Keine Hinweise auf Schädigung der Neugeborenen \\ $\nabla$}

Die Auswertung ergab zunächst, dass 3 Frauen im ersten Trimester, 11 Frauen im zweiten Trimester und 18 Frauen im dritten Trimester über im Median 3 Tage Tamsulosin erhalten hatten, 4 Frauen waren in mehr als einem Trimester behandelt worden.

In der Gruppe der exponierten Schwangeren fanden sich keine Hinweise auf eine vermehrte Schädigung der Neugeborenen. Das durchschnittliche Gestationsalter bei der Geburt betrug 38 Wochen, 6 Kinder kamen vor der 37. Woche zur Welt, im Vergleich zu 12 Kindern in der Kontrollgruppe. Das Geburtsgewicht wurde in allen Fällen als dem Gestationsalter entsprechend beurteilt, Fehl- und Totgeburten traten ebenso wenig auf wie kongenitale Malformationen.

erhalten hatten. Diesen Frauen stellten sie eine im Hinblick auf demografische und klinische Faktoren im Verhältnis 2:1 angepasste Gruppe von Schwangeren gegenüber, die bei der gleichen Symptomatik keine Medikamente zur Steinaustreibung erhalten hatten.

Beurteilt wurden die Häufigkeit von spontanen Fehl- und Frühgeburten, intrauterinem Fruchttod, Kopfumfang der Neugeborenen, kongenitale Fehlbildungen, Ap-
Bei keinem der vorab festgelegten Parameter fanden sich statistisch signifikante Unterschiede zwischen den Gruppen. Allerdings kam es bei 2 Kindern der intrauterin gegenüber Tamsulosin exponierten Gruppe (7\%) zu einem plötzlichen Kindstod (SIDS) vor dem 6. Lebensmonat, in der Kontrollgruppe war das bei keinem Kind der Fall.

\section{Fazit}

Nach diesen Daten gibt es keine Belege dafür, dass die kurzzeitige Gabe von Tamsulosin während der Schwangerschaft den ungeborenen Kindern schadet. Einschränkend gelten die geringe Zahl der Patientinnen und vor allem die geringe Zahl derer, die das Medikament im ersten Trimester erhielten. Das Auftreten von zwei Fällen eines SIDS in der Tamsulosin-Gruppe kann nicht ohne Weiteres auf Tamsulosin zurückgeführt werden, da weitere SIDS-Risikofaktoren unklar sind, u.a. Schlafposition oder Raucher im Haushalt. Trotzdem müssen größere Kohorten die Unbedenklichkeit von Tamsulosin in der Schwangerschaft weiter überprüfen, bevor allgemeine Empfehlungen ausgesprochen werden können.

\section{Dr. Elke Ruchalla, Bad Dürrheim}


Kommentar

\section{Tamsulosin in der Schwangerschaft - sicher, aber sinnvoll?}

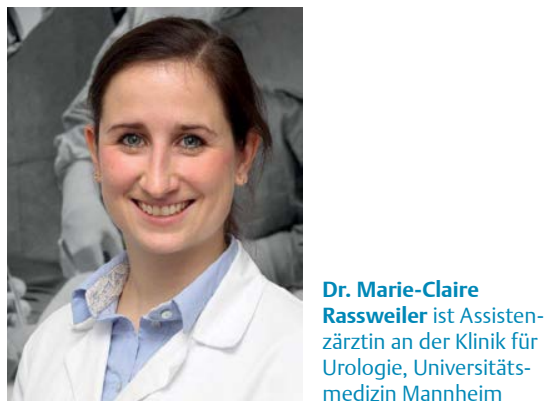

Die Therapie der symptomatischen Urolithiasis in der Schwangerschaft stellt für den behandelnden Urologen eine therapeutische und diagnostische Herausforderung dar. Aufgrund der möglichen teratogenen bzw. mutagenen Risiken sollten auf diagnostische Verfahren mit Strahlenbelastung (Röntgen, CT) verzichtet sowie jegliche medikamentöse Therapie in Abwägung der Beschwerden limitiert verabreicht werden. Eine urologische Intervention sollte ebenfalls sehr zurückhaltend in Erwägung gezogen werden.

In dieser Studie wird erstmals die medikamentöse expulsive Therapie (MET) bei Urolithiasis bei Schwangeren untersucht. Die Studie stellt daher einen ersten Schritt bzgl. einer möglichen erweiterten Therapieempfehlung dar. Es konnte deutlich gezeigt werden, dass durch die Gabe von Tamsulosin keine negativen Einflüsse auf das Baby post partum zu erwarten sind. Zwei beschriebene Fälle von plötzlichem Kindstot in der MET-Gruppe können nicht mit der Gabe des Medikaments assoziiert werden.

Limiationen der Studie:

- Retrospektives Design mit relativ geringer Fallzahl (27 versus 54)

- Fehlende Angaben zu Steinlokalisation und Größe

Diese Limitationen sind durch die Autoren diskutiert worden. Besonders die Steinlokalisation spielt bei dem Einsatz einer Therapie mittels Alphablocker eine entscheidende Rolle. 29\% der Patientinnen beider Gruppen benötigte eine Intervention, sodass schlussendlich 10 von 19 (53\%) in der MET-Gruppe und 11 von 38 (29\%) in der Kontrollgruppe einen spontanen Steinabgang beobachteten. Dies stellt einen Unterschied von 24\% dar. Ohne jegliche Angaben bzgl. der Steingröße und Lokalisation kann diese Anzahl schwer interpretiert werden. Dennoch scheint die Gabe von Tamsulosin die Steinpassage verbessert zu haben.

In aktuellen Studien zur medikamentösen expulsiven Therapie bei Urolithiasis wird besonders die Gabe von Silodosin mit einer besseren Wirksamkeit gegenüber Tamsulosin und Placebo diskutiert. Allerdings konnten diese Erkenntnisse vor allem für distale Harnleitersteine gezeigt werden [1-4]. Daher sollte in zukünftigen Studien der Einsatz von Silodosin bei distalen Harnleitersteinen bei Schwangeren untersucht werden. Da Silodosin nur für Männer zugelassen ist, muss allerdings zuerst geprüft werden, ob ein Risiko für den Fetus besteht. Bei Tamsulosin wurde in dieser Studie bereits eine Einschätzung der FDA (Food and Drug Administration A) als Kategorie-B-Medikament beschrieben. Weitere prospektive Studien müssen durchgeführt werden bis definitive Aussagen bzgl. der Sicherheit und des Nutzens der Gabe von Tamsulosin getroffen werden können. Dennoch stellt diese Studie einen ersten Hinweis auf den möglichen sicheren Einsatz von Tamsulosin in der Schwangerschaft dar.

Fazit

Bei nachgewiesenem distalen Harnleiterstein kann bei Schwangeren die Gabe von Tamsulosin in Absprache mit der Patientin und engmaschigen Kontrollen in Erwägung gezogen werden.

\section{Dr. Marie-Claire Rassweiler, Mannheim}

Literatur

1 Özsoy M, Liatsikos E, Scheffbuch $\mathrm{N}$ et al. Comparison of silodosin to tamsulosin for medical expulsive treatment of ureteral stones: a systematic review and meta analysis. Urolithasis 2016; DOI: 10.1007/s00240016-0872-y

2 Sur RL, Shore N, L'Esperance J et al. Silodosin to Facilitate Passage of Ureteral Stones: A Multi-institutional, Randomized, Doubleblinded, Placebo-controlled Trial. Eur Urol 2015; 67: 959-964

3 Itoh Y, Okada A, Yasui T et al. Efficacy of selective a1 A adrenoceptor antagonist silodo$\sin$ in the medical expulsive therapy for ureteral stones. Int J Urol 2011; 18: 672-674

4 Pickard R, Starr K, MacLennan G et al. Medical expulsive therapy in adults with ureteric colic: a multicentre, randomised, placebocontrolled trial. Lancet 2015; 386: 341-349 